\title{
Accommodating Retrocausality with Free Will
}

\author{
Yakir Aharonov ${ }^{1,2}$, Eliahu Cohen ${ }^{1,3}$ \& Tomer Shushi ${ }^{4}$ \\ 1 School of Physics and Astronomy, Tel Aviv University, Tel Aviv, Israel. E-mail: eliahuco@post.tau.ac.il \\ ${ }^{2}$ Schmid College of Science, Chapman University, Orange, California, USA. E-mail: yakir@post.tau.ac.il \\ ${ }^{3}$ H. H. Wills Physics Laboratory, University of Bristol, Bristol, UK. E-mail: eliahu.cohen@bristol.ac.uk \\ ${ }^{4}$ University of Haifa, Haifa, Israel. E-mail: tomer.shushi@gmail.com
}

Editors: Kunihisa Morita, Danko Georgiev \& Kelvin McQueen

Article history: Submitted on September 22, 2015; Accepted on December 17, 2015; Published on January 11, 2016.

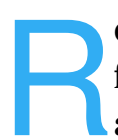

etrocausal models of quantum mechanics add further weight to the conflict between causality and the possible existence of free will. We analyze a simple closed causal loop ensuing from the interaction between two systems with opposing thermodynamic time arrows, such that each system can forecast future events for the other. The loop is avoided by the fact that the choice to abort an event thus forecasted leads to the destruction of the forecaster's past. Physical law therefore enables prophecy of future events only as long as this prophecy is not revealed to a free agent who can otherwise render it false. This resolution is demonstrated on an earlier finding derived from the two-state vector formalism, where a weak measurement's outcome anticipates a future choice, yet this anticipation becomes apparent only after the choice has been actually made. To quantify this assertion, weak information is described in terms of Fisher information. We conclude that an already existing future does not exclude free will nor invoke causal paradoxes. On the quantum level, particles can be thought of as weakly interacting according to their past and future states, but causality remains intact as long as the future is masked by quantum indeterminism. Quanta 2016; 5: 53-60.

\section{Introduction}

Time-symmetric formulations of quantum mechanics are gaining growing interest. Using two boundary conditions rather than the customary one, they offer novel twists to several foundational issues. Such are the WheelerFeynman electromagnetic absorber theory [1], Hoyle and Narlikar's theory of gravitation [2], and Cramer's transactional interpretation [3]. Among these, however, the Aharonov-Bergmann-Lebowitz rule [4] and Aharonov's two-state vector formalism [5] are distinct, in that they even predict some novel effects for a combination of forwards and backwards evolving wave functions. When performing a complete post-selection of the quantum state, otherwise counterfactual questions can be intriguingly answered with regard to the state's previous time evolution.

These advances, however, might seem to come with a price that even for adherents is too heavy, namely, dismissing free will. While quantum indeterminism seemed to offer some liberation from the chains imposed on our choices by classical causality, time-symmetric quantum mechanics somewhat undermines quantum indeterminism, as it renders future boundary conditions the missing source of possible causes. This might eventually reveal

(c) This is an open access article distributed under the terms of the Creative Commons Attribution License CC-BY-3.0, which permits unrestricted use, distribution, and reproduction in any medium, provided the original author and source are credited. 
causality to be just as strict and closed as classical causality. If the future is, in some sense, already there to the point of being causally equal to the past, free will (which is defined in Section 2) might appear to be as illusory as it has appeared within the classical framework. We aim to show this is not necessarily the case. The two-state vector formalism is no worse off than classical physics, or other formulations of quantum mechanics as it pertains to the incorporation of free will. In other words, free will is not precluded even when discussing a quantum world having both past and future boundary.

In this special issue of Quanta, dedicated to Richard Feynman and discussing time-symmetry in quantum mechanics, we examine what might seem to be a problem in these formulations, namely the notion of free will [6]. Discussion of this kind might at first be regarded as philosophical in character, but we hope to formulate the problem rigorously enough to yield nontrivial physical insights.

\section{The Problem}

Following Russell and Deery [6], we propose defining free will as follows. Let a physical system be capable of initiating complex interactions with its environment, gaining information about it and predicting its future states, as well as their effects on the system itself. This grants the system purposeful behavior, which nevertheless fully accords with classical causality. Now let there be more than one course of action that the system can take in response to a certain event, which in turn lead to different future outcomes that the system can predict. Free will then denotes the system's taking one out of various courses of action, independently (at least to some extent) of past restrictions. This definition is very close in spirit to the one employed in [7], i.e. the ability to make choices. It should be emphasized that even in our time-symmetric context, free will means only freedom from the past, not from the future (see also [5]).

In classical physics, conservation laws oblige any event to be strictly determined by earlier causes. In our context, this might apparently leave only one course of action for the system in question, and hence no real choices. When moving to the quantum realm, free will might be recovered [7], but then again, if one adds a final boundary condition to the description of the quantum system, can free will exist? We shall answer both classical and quantum questions on the affirmative, employing statistical and quantum fluctuations, respectively.

In what follows, we analyze a classical causal paradox avoided by the past's instability. We subsequently consider a more acute variant of this paradox and discuss a few possible resolutions. Then we present the quantum counterpart of these two paradoxes where inherent indeterminism saves causality. We show that within the two-state vector formalism, although both future and past states of the system are known, genuine freedom is not necessarily excluded. We then define and quantify weak information that is the kind of information coming from the future that can be encrypted in the past without violating causality.

\section{Interaction between Two Systems with Opposing Time-Arrows}

To demonstrate the possibility of knowing one's future and its consequences, we discuss a highly simplified classical gedanken experiment. Naturally, there are immediate difficulties with such a setup. For example, can two regions in space have opposite time arrows to begin with? Can observers inside them communicate? These and other questions deserve further probing, but we focus here only on what would happen if several conditions are met, rather than whether and how they can be achieved.

Consider, then, a universe comprised of only two closed, non-interacting laboratories located at some distance from one another. Suppose further that their thermodynamic time arrows are opposite to one another, such that each system's future time direction is the other's past. Finally let each laboratory host a free agent, henceforth Alice and Bob, capable of free choice.

It is challenging to create a communication channel between two laboratories of this kind. An exchange of signals is possible in the following form. A light beam is sent from the exterior part of one laboratory to the other's boundary, where a static message is posted. The beam is then reflected back to its origin. If the labs are massive enough, the beam imparts only a negligible momentum transfer.

The gedanken experiment proceeds as follows (Fig. 11): $t_{1}^{(b)}$ : Bob sends a light-beam (red arrow) to Alice's lab. $t_{2}^{(b)}$ : He receives through his returning beam a message from Alice saying: "Let me know if you see this message" (dotted blue world-line).

$t_{3}^{(b)}$ : Bob posts a confirmation saying: "I saw your message" (red world-line).

Then there are the following events in Alice's lab:

$t_{1}^{(a)}$ : Alice sends a light-beam (blue arrow) to Bob's lab.

$t_{2}^{(a)}$ : Alice receives through her returning beam of particles that scattered off Bob's message, i.e. she gets the information from Bob through this beam reflected from Bob's system to her system.

$t_{3}^{(a)}$ : Alice, realizing that this confirmation comes from her future, chooses not to post a message. 


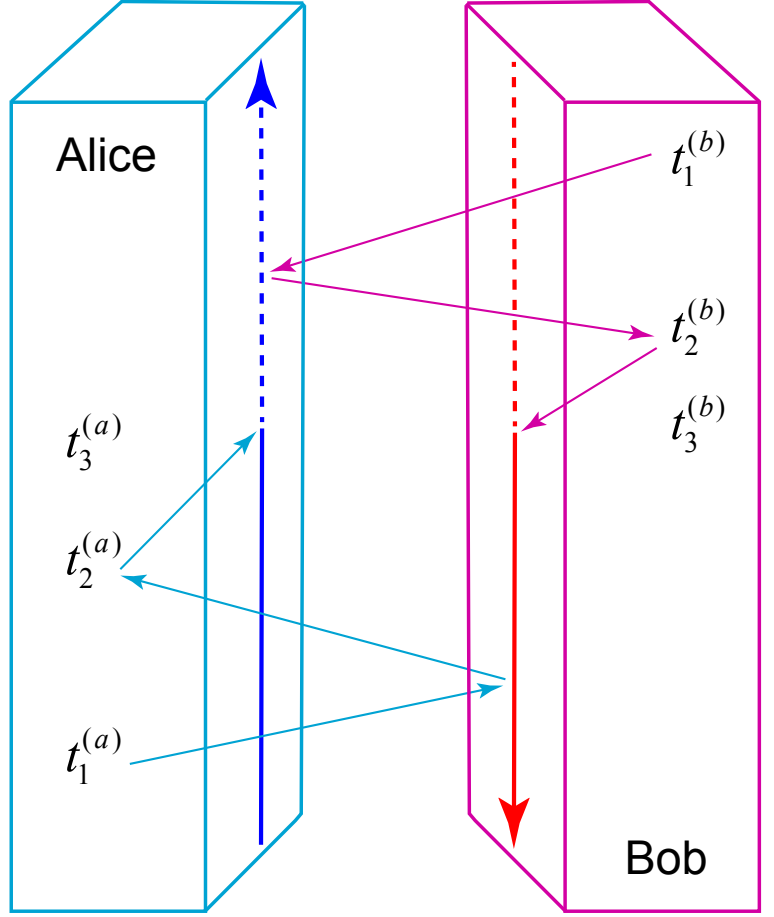

Figure 1: An illustration of the two labs gedanken experiment with two free agents, Alice and Bob.

The Causal Paradox is obvious: The dotted blue worldline represents an absent message. How, then, could Bob reply to a message which was removed before he was supposed to see it?

We note that alongside with this formulation of the paradox, one can equivalently describe the complementary scenario: Bob finds through his returning beam that Alice did not post a message. Therefore, he sends no confirmation, but eventually Alice, having free will, decides to post a message in contrast to Bob's observation.

\section{The Suggested Resolution}

A key element in this causal paradox's resolution is the following well-known fact: Entropy-increasing processes are highly stable, not sensitive to small changes in their initial conditions or their evolution, whereas entropydecreasing processes are extremely vulnerable to any interference.

Our question therefore is: Which time direction is affected by Alice's decision to change the future that has been forecasted by Bob? The simplest and most consistent answer is: Bob's past. Upon Alice's decision to remove her message at $t_{3}^{(a)}$, Bob's prophecy, i.e. the message of Bob to Alice regarding her future choice, turns out to be false. This is clearly inconsistent with his earlier observation of Alice's message, which is understood now to be highly unstable. His observation turns out to be a large (hence very rare) statistical fluctuation.
We can now define the arrow of time of any system as the thermodynamic direction which is stable against changes. While a small change at the large system's present will negligibly affect its future, it can have dramatic effects into its past. Alice's future was coupled in our example to Bob's past. By employing her free will, she could completely alter his previous observations, but the apparent paradox is resolved by taking into account the chaotic nature of the entropy decreasing direction. Indeed, the signals are weak enough, which makes them amenable for this reinterpretation as fluctuations.

\section{A More Acute Paradox}

We shall now discuss an operationally simpler, yet conceptually harder version of the paradox, which emphasizes the role of free will. Let the two labs with opposing time arrows contain two simple machines rather than free agents (see Fig. 2). One machine, A, posts 0 if it receives 0 as an input, and 1 if it receives 1 . The second lab's machine, $\mathrm{B}$, posts 1 if it receives 0 and 0 if it receives 1 . The paradox is as follows: In case A receives 0 from the other lab, it posts 0 . Then $\mathrm{B}$ receives the 0 as an input and posts 1, in contrast to A's earlier input. Alternatively, A receives 1 from $B$, then posts 1 . Then, B receives this 1 and posts 0 , again in contrast to the A's initial input. It follows there are no valid initial conditions for this combined system at a given time.

The resolution may be:

(1) Communication is impossible between two such systems.

(2) The past of both systems is symmetrically unstable.

(3) There must be some stochastic element allowing consistency.

(4) The operations of the two machines must be coordinated.

As explained above, we assume that communication of simple static messages is possible, hence we shall avoid the first option (nevertheless, this paradox could actually suggest that a special communication protocol is needed between two such systems with opposite time arrows). Options (2) and (3) complement each other and resonate with the above notion of free will, as well as with the quantum paradox to be presented below. Naturally, this combination is favored by us. We believe this paradoxical situation could have been avoided if a minor degree of freedom (e.g. at the form of free will) were allowed. In contrast, alternative (4) implies superdeterminism (see for instance [8,9]) or the so called conspiracy between the two machines, which is philosophically disturbing (at least in our view), negating free will altogether. 

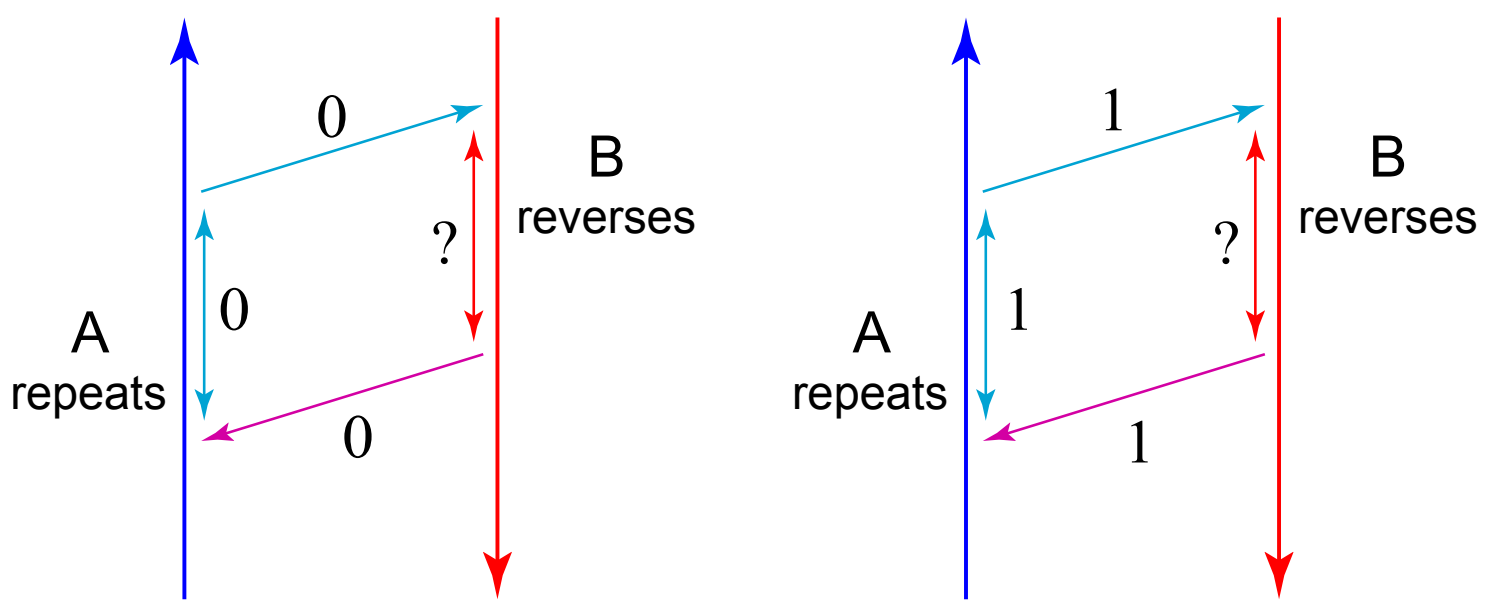

Figure 2: An illustration of the two machines gedanken experiment. Machine A posts 0 (1) if it receives 0 (1), whereas machine $B$ posts $0(1)$ if it receives $1(0)$. The paradox is symmetric, but for simplicity it is shown to reside on the B side.

\section{Going Quantum: The Two-State Vector Formalism and Weak Measurements}

The possibility for resolving the above problem on classical grounds encourages seeking more interesting avenues at the quantum level. Indeed a similar resolution will be offered, namely the possibility of re-interpreting the past. However, the basic concept on which the resolution relies shifts from thermodynamic to quantum fluctuations which are more suitable for describing small microscopic systems. This is where time-symmetric quantum causality comes in most naturally.

The two-state vector formalism is a time-symmetric formulation of quantum mechanics employing in addition to the forward evolving wave function (pre-selected state) also a backwards evolving wave function (post-selected state). This combination gives rise to the two-state-vector, which provides a richer notion of quantum reality between two projective measurements. This world-view has produced several predictions, so far well verified by weak measurements [10-13] which delicately gather information about the quantum state without collapsing it, and thus do not change to post-selection probability.

In an earlier work [13] the following gedanken experiment was proposed. A large ensemble of $N$ spins is prepared in Einstein-Podolsky-Rosen state. Each particle in every pair is weakly measured along the three Bell orientations, before being strongly projected along one of them. As was shown, each weak measurement only slightly disturbs the state and hence the well-known non-local correlations between the strong outcomes are maintained in this experiment. It should be noted that in return each weak measurement provides only a negligible amount of information (to be quantified in Section 7).
However, since all the weak outcomes were classically recorded, upon slicing them according to the projective outcomes, one finds in retrospect, with extreme accuracy, the weak values corresponding to all Bell orientations (not only the ones eventually chosen for the projective measurement). The question is then, how could the values reside in the weak data prior to the final Bell measurements which demonstrated almost perfect non-local correlations? Bell's proof certainly forbids them to be prepared in such a way so the two-state vector formalism answer would be that they came from the future! The important point in this retrocausal interpretation is the weak values could be there, that is, could had causal effect on the pointer's shift, without forcing a specific future outcome.

The resolution is therefore simple: quantum indeterminacy guarantees that, should someone try to abort a future event about which they have received a prophecy, that prophecy would turn out to be a mere error.

Therefore, even in the two-state vector formalism where present is determined by both past and future events, the quantum indeterminism enables free will.

Naturally, more mundane explanations ought to be considered before concluding that results of weak measurement contain information regarding a future event. By normal causality, it should be Alice's measurements which affected Bob's, rather than vice versa. Perhaps, for example, some subtle bias induced by her weak measurements affected his later strong ones.

Such a past-to-future effect is considerably strained by the following question: How robust is the alleged bias introduced by the weak measurements? If it is robust enough to oblige the strong measurements, then it is equivalent to full collapse, namely the very local hidden variables already ruled out by Bell's inequality. This is 
clearly not the case: weakly measured particles remain nearly fully entangled. But then, even the weakest bias, as long as it is expected to show up over a sufficiently large $N$, is ruled out of the same grounds. The weak bias alternative is ruled out also by the robust correlations predicted between all same-spin measurements, whether weak or strong.

Can Alice predict Bob's outcomes on the basis of her own data? To do that, she must feed all her rows of outcomes into a computer that searches for a possible series of spin-orientation choices plus measurement outcomes, such that, when she slices her rows accordingly, she will get the complex pattern of correlations described above.

The number of such possible sequences that she gets from her computation is $\left(\begin{array}{c}N \\ N / 2\end{array}\right) \propto \frac{2^{N}}{\sqrt{N}}$. Each such sequence enables her to slice each of her rows into two $N / 2$ halves and get the above correlations between her weak measurements and the predicted strong measurements. The distribution of the results is a Gaussian with $\lambda \sqrt{N} / 2$ expectation and $\delta \sqrt{N} / 2$ standard deviation, so a $\delta$ shift in one of the results, or even in $\sqrt{N}$ of them, is very probable. Hence, even if Alice computes all Bob's possible future choices, she still cannot tell which choice he will take, because there are many similar subsets giving roughly the same value. Also, as Aharonov et al. pointed out in [13], when Alice finds a subset with a significant deviation from the expected 50\%-50\% distribution, its origin is much more likely, upon a real measurement by Bob, to turn out to be a measurement error than a genuine physical value. Obviously, then, present data is insufficient to predict the future choice.

\section{The Strength of Information Transmission}

The information transmission between Alice and Bob can be categorized into two different types with different strength:

Strong information: This type describes the information that, in general, has the potential to interfere with Alice's free choice. This is the classical kind of information transmitted in the first gedanken experiment.

Weak information: In this case the information that Bob sends to Alice will not, in any circumstance, interfere with Alice's free choice because it is buried much below the quantum uncertainty level.

While the strong information transmission was discussed in Sections 3 and 4 and was shown to cast instability into Bob's past, it seems the weak information notion should be further explained and quantified.

We now understand that weak information represents information that does not actively interfere with the Alice's and Bob's systems. Therefore, weak information can be described employing weak measurement outcomes since individually they only provide very partial information that does not interfere with neither Alice's nor Bob's system consistency. Similarly, strong information is related to projective measurement outcomes since they do disturb the systems and provide definite results.

To create a clear distinction between the two kinds of information, we shall discuss a simple thought experiment. Suppose Alice has a spin she wants to measure. To do that, she will use a Stern-Gerlach magnet with a nonhomogeneous magnetic field along some direction. Bob, having an opposite time arrow, already knows that Alice will choose the $z$-axis and will find an $u p$ outcome. If Bob sends this strong information to Alice, she may choose the $y$-axis instead and find a down outcome, reproducing the paradoxical situation discussed above. However, if Bob only tells her that she will find an up result along some direction, no causal paradox will ensue (see also [14]. This is the kind of weak information which does not clash with Alice's free will nor with Bob's history.

\section{Fisher Information for Strong and Weak measurements}

Fisher information is a tool to quantify the hidden information in a random variable $Q$ regarding a parameter it depends on. Using Fisher information we can now quantitatively define the strong and weak information concepts that were qualitatively introduced in Section 7 .

Suppose that there is an unknown parameter $\theta$ which we want to estimate (for example, $\theta$ can stand for the relative phase between two superposed wave-packets). We define a density function $f$ of $Q$ and another auxiliary parameter $\Delta$ which describes the type of information, strong or weak. In probabilistic terms, it is called the scale parameter of $Q$. In this case, Fisher information as a function of $\Delta, I_{\Delta}(\theta)$, is given by

$$
I_{\Delta}(\theta):=E\left(\left[\frac{\partial}{\partial \theta} \ln f(\Delta Q ; \theta)\right]^{2} \mid \theta\right) .
$$

It can be easily shown that $I_{\Delta}(\theta)$ is in fact the product of $\Delta^{-1}$ and $I(\theta)$ :

$$
\begin{aligned}
I_{\Delta}(\theta) & =\int\left[\frac{\partial}{\partial \theta} \ln f(\Delta Q ; \theta)\right]^{2} f(\Delta Q ; \theta) d Q \\
& =\frac{1}{\Delta} \int\left[\frac{\partial}{\partial \theta} \ln f(Q ; \theta)\right]^{2} f(Q ; \theta) d Q \\
& =\Delta^{-1} I(\theta) .
\end{aligned}
$$


Now, in case of $\Delta \rightarrow 0$, we find

$$
\lim _{\Delta \rightarrow 0} I_{\Delta}(\theta)=\lim _{\Delta \rightarrow 0} \Delta^{-1} I(\theta)=\infty
$$

hence we conclude that $\Delta \rightarrow 0$ indicates strong information.

The opposite case of $\Delta \rightarrow \infty$ leads to

$$
\lim _{\Delta \rightarrow \infty} I_{\Delta}(\theta)=\lim _{\Delta \rightarrow \infty} \Delta^{-1} I(\theta)=0
$$

which implies weak information, so for sufficient large value of $\Delta$, weak information is described by a negligible Fisher information.

Let us now demonstrate this concept. Suppose that $\theta$ is the relative phase between two superposed wavepackets, which we want to measure in some interference experiment. Let us assume that the interference pattern is detected via some coupling $1 / \Delta$ to a measuring pointer. If our estimation for the relative phase is described by a Gaussian random variable $Q$, then the density function of $Q$ will be

$$
f(Q ; \theta)=\frac{1}{\sqrt{2 \pi} \theta} \exp \left(-\frac{1}{2 \theta^{2}} Q^{2}\right) .
$$

Depending on the coupling strength, the Fisher information will be

$$
I_{\Delta}(\theta)=\Delta^{-1} \theta^{-2} .
$$

\section{Cryptography Can Protect Causality}

Weak or encrypted information can be used for communication between future and past in a causality preserving manner thanks to quantum indeterminism. The main idea behind this type of communication is quantum cryptography [15]. Suppose Bob somehow knows what Alice will choose in the future. He uses a quantum cryptography scheme to encode Alice's future choice and gives her the encrypted prophecy. However, he does not share with her the key to decode this revelation until she actually makes her choice. In this case, similarly to the example in Section 6, both Bob's past and Alice's future are secured. Due to quantum indeterminism, Alice still has free will.

For example, in the BB84 scheme [16, 17], even though Alice and Bob communicate through a public channel, their secret key is secured due to another form of quantum indeterminism, namely, that non-orthogonal states are indistinguishable. This means that even if the generated string contains information regarding Eve's future, it will not create a causal paradox.

\section{A Few Alternatives}

In addition to the proposed resolution for the above paradoxes, there exist some other well-known possibilities. The parallel universe resolution suggests that if one goes back in time and kills his grandfather he actually does it in a parallel universe and therefore he does not interfere with the laws of nature [18, 19]. A different approach to solve this is by postulating another time dimension in which such disagreements can be solved before being recorded in our history [20,21].

These two resolutions clearly lack simplicity and oblige an excessive ontology to our existing theories. Moreover, detailed work is needed to refute each and every paradox.

Therefore, bearing in mind Occam's razor as a tool for denying complex theories, it seems these alternative solutions are unfavorable.

Another solution simply dictates that one cannot create paradoxes in the universe and therefore cannot, for instance, kill his grandfather. This approach implies a universe guided by global consistency condition such as in [22,23] and was shown to naturally arise in postselected closed timelike curves [24].

\section{Free will and Becoming}

Classical physics treats time as a purely geometrical ingredient of the universe, alongside the three spatial dimensions. Against the perfect logical rigor and experimental support that make relativity so powerful, many physicists find the block universe picture emerging from it manifestly awkward. In fact, the very notion of space-time implies that, just as all locations have the same degree of reality in space, so do all past, present, and future moments exist along the temporal dimension without any moment being unique as the privileged now.

Against this mainstream view, there are alternative accounts [25]. They suspect that, if we experience time so differently from space, this difference may be objective. They provide some models to capture this notion of dynamic time.

Bob's access to Alice's future in the classical gedanken experiment above and the double boundary condition on the wave function proposed by the two-state vector formalism may seem at first sight to resonate with a block universe approach. However, as we have just seen, statistical and quantum fluctuations may provide us with freedom to define the present. As was shown in Sections 4 and 5, this freedom, and also the notion of becoming, is subjective and system-dependent. 
Within the two-state vector formalism, while both backward and forward states evolve deterministically, they have limited physical significance on their own - physical reality is created by the product of the causal chains extending in both temporal directions. The past does not determine the future, yet the future is set, and only together do they form the present. However, the existence of a future boundary condition, and its deterministic effect, do not deny our freedom of choice. It is allowed due to the inaccessibility of the data (which is a requirement of causality, as discussed in Section 57. Examining the concept of free will from a physical point of view, we find that it must contain at least partial freedom from past causal constraints, and such freedom is duly manifested in the two-state vector formalism, where a juxtaposition of freedom and determinacy is epitomized.

\section{Conclusions}

We examined the possibility of free will in a retrocausal theory. Closed causal loops, which arise due to the interaction between two systems with opposing time arrows were discussed. The suggested resolution of the ensuing paradoxes relies on the thermodynamic instability of the past.

Moving to the quantum realm, a similar paradox can be solved via the quantum indeterminism, which is understood to protect free will. This resonates with previous findings of Georgiev [7]. Furthermore, we discussed the strength of information transmission, where the terms strong and weak are related to strong (projective) and weak values, respectively. When information about a future event is buried under quantum indeterminism it cannot violate free will. Similarly, encrypted information, such as the one available through weak measurements, does not violate causality. The existence of free will in these time symmetric models was conjectured to resonate with a dynamical notion of time.

\section{Acknowledgements}

We thank Avshalom C. Elitzur and Nissan Itzhaki for very helpful discussions. Yakir Aharonov and Eliahu Cohen acknowledge support of the Israel Science Foundation Grant No. 1311/14. Yakir Aharonov acknowledges support from ICORE Excellence Center "Circle of Light". Eliahu Cohen was also supported by ERC-AD NLST.

\section{References}

[1] Wheeler JA, Feynman RP. Interaction with the absorber as the mechanism of radiation. Reviews of Modern Physics 1945; 17 (2-3): 157-181. doi: 10.1103/RevModPhys.17.157

[2] Hoyle F, Narlikar JV. A new theory of gravitation. Proceedings of the Royal Society of London A: Mathematical, Physical and Engineering Sciences 1964; 282 (1389): 191-207. doi : 10. 1098/rspa. 1964.0227

[3] Cramer JG. The transactional interpretation of quantum mechanics. Reviews of Modern Physics 1986; 58 (3): 647-687. doi : 10. 1103/RevModPhys . 58 . 647

[4] Aharonov Y, Bergmann PG, Lebowitz JL. Time symmetry in the quantum process of measurement. Physical Review B 1964; 134 (6): 1410-1416. doi : 10.1103/PhysRev. 134.B1410

[5] Aharonov Y, Cohen E, Gruss E, Landsberger T. Measurement and collapse within the two-state vector formalism. Quantum Studies: Mathematics and Foundations 2014; 1 (1-2): 133-146. doi : 10. 1007/s40509-014-0011-9

[6] Russell P, Deery O. The Philosophy of Free Will: Essential Readings from the Contemporary Debates. New York: Oxford University Press, 2013.

[7] Georgiev D. Quantum no-go theorems and consciousness. Axiomathes 2013; 23 (4): 683-695. doi : $10.1007 / \mathrm{s} 10516-012-9204-1$

[8] 't Hooft G. The free-will postulate in quantum mechanics. 2007: arXiv:quant-ph/0701097

[9] 't Hooft G. The cellular automaton interpretation of quantum mechanics. A view on the quantum nature of our universe, compulsory or impossible? 2014: arXiv: 1405.1548

[10] Aharonov Y, Albert DZ, Vaidman L. How the result of a measurement of a component of the spin of a spin- $\frac{1}{2}$ particle can turn out to be 100 . Physical Review Letters 1988; 60 (14): 1351-1354. doi: 10.1103/PhysRevLett. 60.1351

[11] Tamir B, Cohen E. Introduction to weak measurements and weak values. Quanta 2013; 2 (1): 7-17. doi : 10.12743/quanta.v2i1. 14 
[12] Aharonov Y, Cohen E, Elitzur AC. Foundations and applications of weak quantum measurements. Physical Review A 2014; 89 (5): 052105. doi: 10.1103/PhysRevA.89.052105

[13] Aharonov Y, Cohen E, Elitzur AC. Can a future choice affect a past measurement's outcome? Annals of Physics 2015; 355: 258-268. arXiv: 1206. 6224, doi: $10.1016 / j$.aop.2015.02.020

[14] Elitzur AC, Cohen E, Shushi T. The too-late-choice experiment: Bell's proof within a setting where the nonlocal effect's target is an earlier event. 2015; arXiv: 1512.08275

[15] Gisin N, Ribordy G, Tittel W, Zbinden H. Quantum cryptography. Reviews of Modern Physics 2002; 74 (1): 145-195. arXiv:quant-ph/0101098, doi: 10.1103/RevModPhys.74.145

[16] Bennett CH, Brassard G. Quantum cryptography: Public key distribution and coin tossing. Proceedings of the IEEE International Conference on Computers, Systems and Signal Processing, Bangalore, India, December 10-12, 1984, pp. 175-179.

[17] Bennett CH, Brassard G. Quantum cryptography: Public key distribution and coin tossing. Theoretical Computer Science 2014; 560 (1): 7-11. doi: 10 . $1016 /$ j.tcs.2014.05.025

[18] Bousso R, Susskind L. Multiverse interpretation of quantum mechanics. Physical Review D 2012; 85 (4): 045007. arXiv: 1105.3796, doi:10.1103/ PhysRevD.85.045007

[19] Deutsch D. The structure of the multiverse. Proceedings of the Royal Society of London A: Mathematical, Physical and Engineering Sciences 2002; 458 (2028): 2911-2923. arXiv:quant-ph/0104033. doi:10.1098/rspa.2002.1015
[20] Bars I. Survey of two-time physics. Classical and Quantum Gravity 2001; 18 (16): 31133130. arXiv:hep-th/0008164, doi:10.1088/ 0264-9381/18/16/303

[21] Bars I, Terning J. Two-time physics. In: Extra Dimensions in Space and Time. Multiversal Journeys, Nekoogar F (editor), New York: Springer, 2010, pp. 67-87. doi:10.1007/978-0-387-77638-5_ 7

[22] Carlini A, Frolov VP, Mensky MB, Novikov ID, Soleng HH. Time machines: the principle of selfconsistency as a consequence of the principle of minimal action. International Journal of Modern Physics D 1995; 4 (5): 557-580. arXiv:gr-qc/ 9506087, doi : 10.1142/S0218271895000399

[23] Novikov ID. Time machine and self-consistent evolution in problems with self-interaction. Physical Review D 1992; 45 (6): 1989-1994. doi : 10.1103/ PhysRevD.45.1989

[24] Lloyd S, Maccone L, Garcia-Patron R, Giovannetti V, Shikano Y. Quantum mechanics of time travel through post-selected teleportation. Physical Review D 2011; 84 (2): 025007. arXiv: 1007.2615, doi:10.1103/PhysRevD.84.025007 http://hdl.handle.net/1721.1/66971

[25] Aharonov Y, Popescu S, Tollaksen J. Each instant of time a new universe. In: Quantum Theory: A Two-Time Success Story. Yakir Aharonov Festschrift. Struppa DC, Tollaksen JM (editors), Milan: Springer, 2014, pp. 21-36. doi:10.1007/ 978-88-470-5217-8_3 